\title{
In-line Variable Optical Attenuator Based on the Bending of the Tapered Single Mode Fiber
}

\author{
Kwang Taek Kim, Ji Hoon Kang, and Seung Hwangbo \\ Department of Optoelectronics, Honam University, Gwangju 506-714, Korea \\ Kiegon $1 \mathrm{~m}^{*}$ \\ Department of Physics, Chonnam National University, Gwangju 500-757, Korea
}

(Received May 20, 2009 : revised July 3, 2009 : accepted July 6, 2009)

\begin{abstract}
We propose a simple in-line variable optical attenuator (VOA) based on the bending effect of tapered single mode fibers. The influence of the taper structure and the reflective index of the external medium surrounding the taper region on the bending loss of the tapered fiber have been investigated experimentally. An attenuation range exceeding $35 \mathrm{~dB}$ and a very low excess loss of $<0.2 \mathrm{~dB}$ at $1550 \mathrm{~nm}$ were achieved. The measured polarization dependent loss of the present VOA at the attenuation level of $10 \mathrm{~dB}, 20 \mathrm{~dB}$, and $30 \mathrm{~dB}$ were $0.1 \mathrm{~dB}, 0.2 \mathrm{~dB}$, and $0.5 \mathrm{~dB}$, respectively.
\end{abstract}

Keywords: Variable optical attenuator, Tapered single mode fiber, Bending loss

OCIS codes: (060.0060) Fiber optics and optical communications; (060.2280) Fiber design and fabrication; (060.2340) Fiber optics components; (060.2430) Fibers, single-mode

\section{INTRODUCTION}

Variable optical attenuators (VOAs) are used widely in metro and access optical networks to control the optical power level of each channel at the cross connect points and add-drop nodes. Optical receivers often require VOAs to maintain their optimal input power level. Variable attenuators using integrated waveguides [1-3] and MEMS [4-6] have been reported. Robustness, compact size and low cost are required for VOAs in an optical network. An in-line configuration can solve the interface problem between a VOA and a single mode fiber, and ensure low insertion loss, negligible back reflection, good mechanical reliability and a simple fabrication process.

Tapered fibers are used in a variety of fiber optic communication devices or sensors [7-11]. The evanescent wave expands in the waist region of a tapered single mode fiber and the core mode can be coupled easily with radiation modes. The investigation of bending effect of standard single mode fiber [12] and tapered fiber [13-14] and its applications as a pressure sensor [15] and an optical modulator [16] have been reported.

*Corresponding author: kgim@chonnam.ac.kr
This study employed the bend sensitive property of the tapered single mode fiber to produce a simple VOA with high attenuation capability. A similar VOA based on a bend sensitive fiber whose core structure is different from that of the standard single mode fiber has also been reported. However, the mode mismatching between the bend sensitive fiber and the standard single mode fiber caused a considerable insertion loss [17]. For a tapered single mode fiber with its core size reduced, the transverse distribution of the propagation mode spreads further into the cladding region, resulting in a more weakly guided light beam. The throughput of the core mode becomes quite sensitive to bending, which can be utilized in high precision attenuators in a controlled manner. In this study we have examined the influence of taper structure and the refractive index of the external medium surrounding the taper region on the bending loss and the transmission loss. The device structure appropriate for both high attenuation range and small insertion loss has been presented. The attenuation of the tapered fiber was controlled by adjusting its bending radius. It is expected that the simple structure and easy fabrication process of the proposed VOA may be useful for the optical metro and access networks. 


\section{DESIGN CONSIDERATION}

Fig. 1 shows a schematic diagram of the tapered fiber and proposed VOA. The tapered section can be divided into two transition regions and a waist region with a uniform radius, as shown in Fig. 1(a). The radius of the fiber core decreases from $\mathrm{a}_{0}$ to $\mathrm{a}_{\mathrm{w}}$ in the leading transition region. The transverse distribution of the propagation mode expanded adiabatically according to mode evolution effect. The characteristics of the tapered fiber are dependent on the tapering ratio $\left(\mathrm{a}_{0} / \mathrm{a}_{\mathrm{w}}\right)$, length of the transition region $\left(\mathrm{L}_{\mathrm{t}}\right)$ and length of the waist region $\left(\mathrm{L}_{\mathrm{w}}\right)$, as shown in the Fig. 1(a). The tapered fiber can be bent with the help of a micrometer slide as shown in Fig. 1(b). The attenuation of the input optical power can be controlled by the bending radius of the fiber, which is in turn adjusted using a micrometer slide.

The bending loss per unit length of step index single mode fibers can be expressed as follows [12]:

$$
\begin{aligned}
& \alpha=\frac{1}{2}\left(\frac{\pi}{a W^{3}}\right)^{1 / 2}\left(\frac{U}{V K_{1}(W)}\right)^{2} \exp (-D \cdot R) \cdot R^{-1 / 2} \\
& D=\frac{4 \Delta W^{3}}{3 a V^{2}}
\end{aligned}
$$

where $a, R$ and $\Delta$ are the core radius, bending radius, and core-cladding refractive index difference, respectively. $K_{1}$ is a modified Hankel function, $U$ and $W$ are the

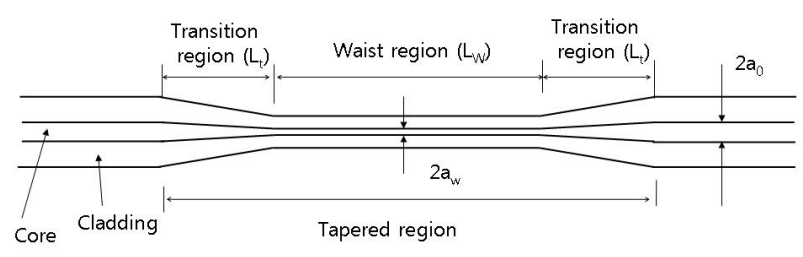

(a)

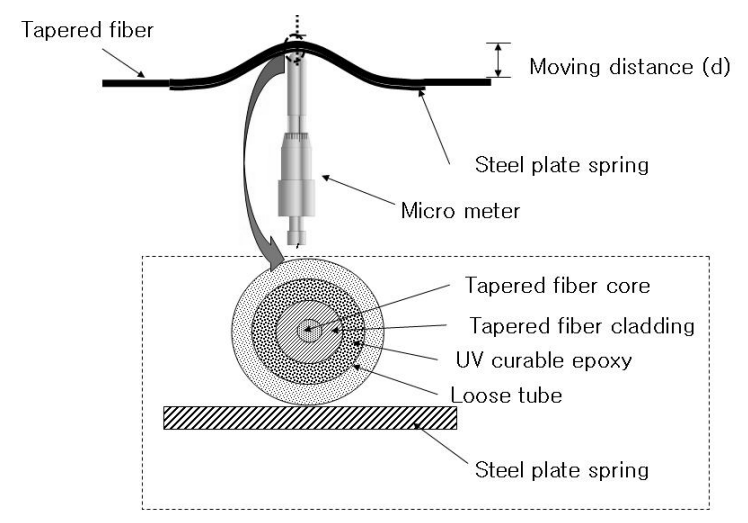

(b)

FIG. 1. Schematic diagram of the proposed variable optical attenuator; (a) the tapered single mode fiber, (b) the bending with a micrometer. transverse propagation constants in the core and cladding, respectively, and $V$ is the normalized frequency.

Fig. 2 shows the calculated bending loss for a step index single mode fiber at $1550 \mathrm{~nm}$. The refractive indices of the cladding and core were assumed to be 1.444 and 1.449 , respectively. The bending loss increases steeply with decreasing bending radius, and is quite sensitive to the core size when the other parameters are fixed.

A tapered single mode fiber behaves like a multimode fiber when the refractive index of external medium surrounding the tapered region is less than that of the fiber cladding. The mode coupling among the core mode and cladding modes occurs as the tapered fiber is bent and the bending loss oscillates with change of bending curvature $[13,14]$. As the diameter of the core and the cladding decrease in a tapering process, the cladding takes up the role of the core and the low index external medium plays the role of the cladding in turn so that higher order modes can be guided in the tapered region and the bending loss exhibits oscillatory behavior as bending radius changes. In order to avoid oscillation of bending loss, the tapered fiber was recoated with a transparent epoxy whose refractive index was equal to or higher than that of the cladding as shown in Fig. 1(b). This epoxy layer not only suppresses the higher order modes but also reinforces the mechanical strength of the tapered fiber.

The beam propagation method has been used to calculate the transmission loss and the bending effect of tapered fibers. The refractive indices of the cladding and the core were 1.444 and 1.449 , respectively. Fig. 3 shows the calculated mode propagation and the excess loss of tapered fiber without bending. For a tapered fiber with its diameter reduced to $4.0 \mu \mathrm{m}$, the optical beam propagates without significant loss as shown in Fig. 3(a). However, the loss becomes excessive when the diameter is reduced to $3.0 \mu \mathrm{m}$ as shown in Fig. 3(b). The transmission loss is mainly due to the insufficient

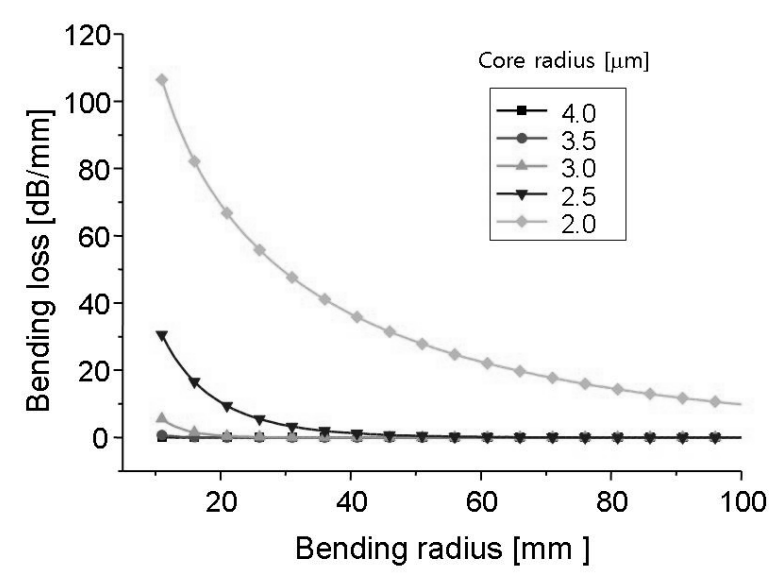

FIG. 2. The bending radius dependence of the bending loss and the effect of the core size on the bending loss. 


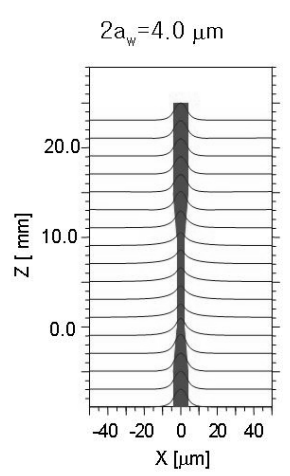

(a)

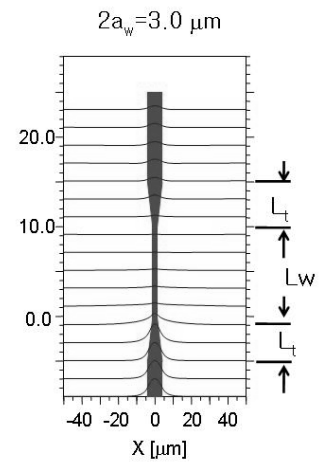

(b)

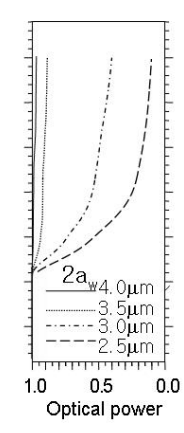

(c)
FIG. 3. Simulation results of a tapered fiber without bending; (a) mode propagation of tapered fiber with 2.0 $\mu \mathrm{m}$ core radius at the waist, (b) mode propagation of tapered fiber with $1.5 \mu \mathrm{m}$ core radius at the waist, (c) optical power variation along the tapered fiber under four different tapering conditions. (a)

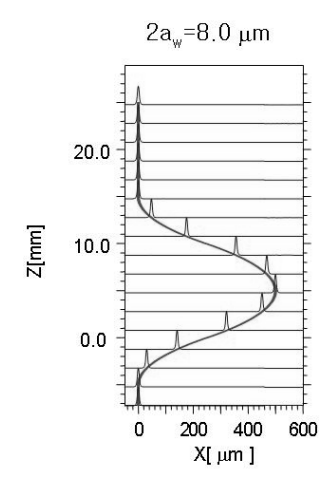

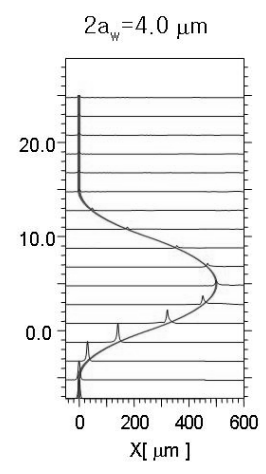

(b)

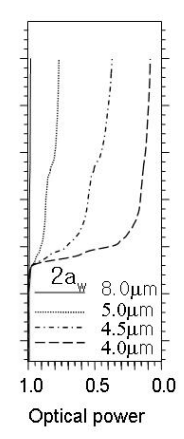

(c)
FIG. 4. Simulation results of tapered fiber with bending; (a) mode propagation of non tapered fiber, (b) mode propagation of tapered fiber with $2.0 \mu \mathrm{m}$ core radius at the waist, (c) optical power variation along the tapered fiber for four different tapering conditions.

length of the tapered section of fiber for an adiabatic mode conversion. Smaller core size requires a longer transition length for low transmission loss. For example, a tapered single mode fiber with $3 \mu \mathrm{m}$ core diameter requires $20 \mathrm{~mm}$ long length of transition region for a transmission higher than $90 \%$. Fig. 3(c) shows that the optical power decreases along the tapered fibers of different core diameters. There can be a practical limit in reducing the core size of tapered fiber for a VOA. Excess transmission loss may occur in conjunction with the expanded mode profile and the beam power leaks into the external medium.

Fig. 4 shows the mode propagation and the bending loss of the bent fibers with or without a taper. The optical mode passes through the bent region of the uniform core fiber without noticeable loss, as shown in
Fig. 4(a). In contrast, the bending loss is significantly high at the bent region of the tapered fiber as shown in Fig. 4(b). Calculated optical power along the tapered fiber for four different core diameters at the waist is shown in Fig. 4(c). The tapering ratio $\left(\mathrm{a}_{0} / \mathrm{a}_{\mathrm{w}}\right)$ must be selected carefully in order to satisfy the requirement of low excess loss as well as high bending loss. From the calculation results shown in Fig. 3 and Fig. 4, the optimized tapering ratio is approximately 2.0 for a standard single mode fiber in telecommunications. In the above simulation, we assumed that the cladding diameter was infinite. For an actual device, the cladding diameter is finite and the bending loss is affected by the refractive index of the external medium covering the tapered region, which is the case described in the next section.

\section{EXPERIMENTS AND ANALYSIS}

A standard single mode fiber (SMF 28) was used in this experiment. The fiber was tapered using the flame brushing method [18-20]. After removing the coated layer from a $20 \mathrm{~mm}$ length of the fiber section, the fiber was brushed along the $10 \mathrm{~mm}$ length at a constant speed with the mixed oxygen and LPG flames from two micro torches and was pulled along its length to be tapered. Three different tapered fibers were prepared and the increased amount of length $\Delta \ell$ for the samples $\mathrm{A}, \mathrm{B}$, and $\mathrm{C}$ was $5.0,7.5$, and $10 \mathrm{~mm}$, respectively, as shown in Fig. 5. The combined microscopy images in Fig. 5 were obtained by joining many photographs of an area approximately $100 \mu \mathrm{m}$ in length taken every $1.0 \mathrm{~mm}$ step along the fiber in an effort to emphasize the tapered shape. The outer diameter of the fiber cladding at the tapered waist for samples A, B, and $\mathrm{C}$ was $90.8,79.6$, and $68.4 \mu \mathrm{m}$, respectively. When the sample was not bent, the optical power transmitted through the tapered fibers was not changed when they were immersed in the high index liquid, which suggests that the evanescent field of the propagation mode was not extended into the external medium even around the waist region.

The influence of the refractive index of the external medium on the bending loss was examined for the sample $\mathrm{C}$ tapered fiber. In Fig. 6, the air, water, and two different index fluids were used as the external medium. When the refractive indices of the external medium were less then that of fiber cladding (1.444), the bending loss curve oscillates as the bending curvature increases. There is almost no oscillatory behavior in the bending loss for the index liquid of its refractive index (1.445).

The tapered fiber was recoated with an UV curable epoxy and was inserted into a loose tubing of $0.9 \mathrm{~mm}$ inside diameter and the tubing was filled with UV curable epoxy (SK-9) of its refractive index of 1.48 in 

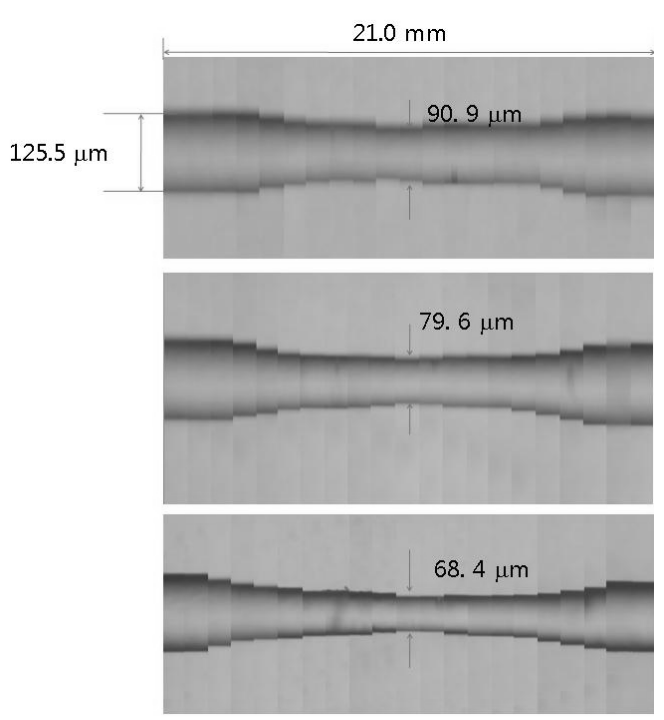

FIG. 5. Microscope pictures of fabricated tapered fiber; (a) sample A, (b) sample B, (c) sample C.

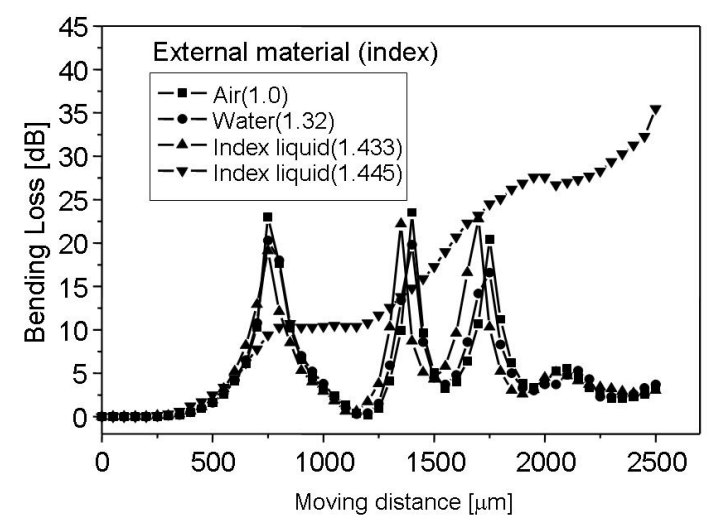

FIG. 6. Measured bending loss of a tapered fiber surround by different external medium.

the capillary effect. The epoxy plays the role of an optical sink in such a way that the optical power leaks into the epoxy when the tapered fiber is bent. The coated tapered fiber was then attached to a $0.11 \mathrm{~mm}$ thick steel plate spring and packaged into a jig platform equipped with a micrometer, as shown in Fig. 7. The bending radius of curvature of the tapered fiber was controlled by moving the micrometer tip. The working distance of the micrometer was $2.5 \mathrm{~mm}$ and the corresponding bending radius of the steel plate spring was approximately $17 \mathrm{~mm}$. We have not performed a durability test for the present device. However, the tapered fiber packaged in a jig with stainless steel plate did not show any fatigue during the whole experiment period.

The measured bending loss was shown in Fig. 8. The non tapered fiber showed negligible bending loss over the entire range of working distances of the installed micrometer. Its attenuation was less than $0.24 \mathrm{~dB}$. The maximum attenuation of $31 \mathrm{~dB}$ was obtained from the

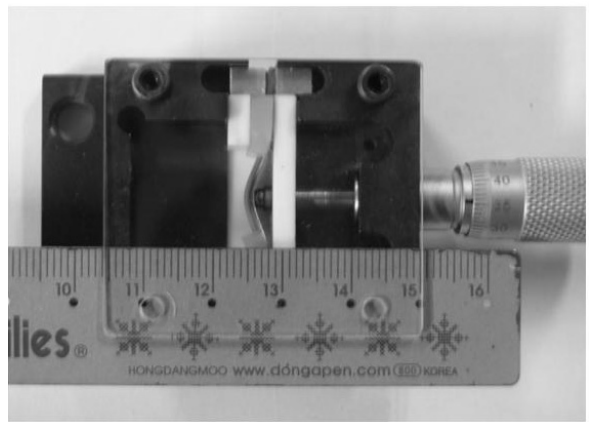

FIG. 7. The jig with micrometer for fiber bending.

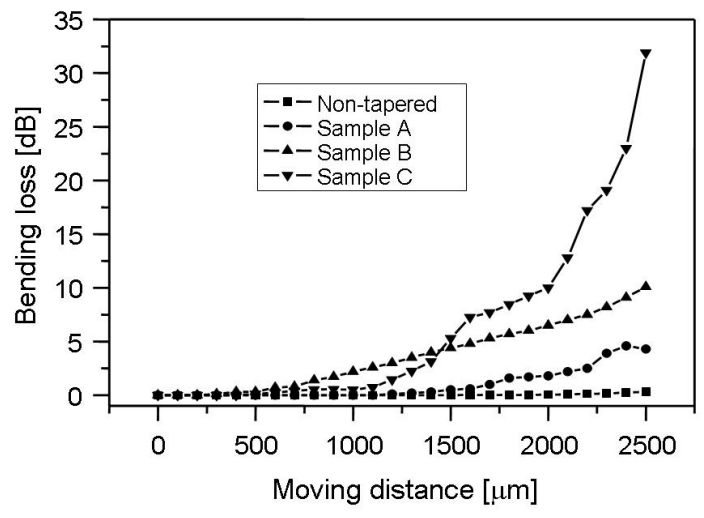

FIG. 8. Measured bending loss of recoated three tapered fibers and a non tapered fiber.

sample $\mathrm{C}$, the most highly tapered fiber. The attenuation range could be adjusted by controlling the tapering condition during the fabrication process. The structures appeared in the curves in Fig. 8 may as well be due to the index mismatching between the fiber cladding and the epoxy layer. The refractive index of the epoxy surrounding the fiber cladding is slightly higher than that of fiber cladding by 0.036 . The bending loss is expected to vary in a more monotonic fashion if a more index matched epoxy is employed. The insertion loss of VOAs fabricated in this study was less than $0.2 \mathrm{~dB}$, which was measured with the unbent tapered fiber. The measured polarization dependent loss for the sample $\mathrm{C}$ was $0.1 \mathrm{~dB}, 0.4 \mathrm{~dB}$, and $0.5 \mathrm{~dB}$ at the attenuation level of $10 \mathrm{~dB}, 20 \mathrm{~dB}$, and $30 \mathrm{~dB}$ respectively.

The key features of the present VOA include the simple structure, easy fabrication procedure, and high performance due to the in-line device configuration. By employing tapered fibers, the desired attenuation can be obtained easily without excessive bending, which is an essential property of a compact mechanical type VOA.

\section{CONCLUSION}

This paper reported an in-line optical variable attenuator based on the tapered single mode fiber. The 
effect of tapering on both the excess and bending losses was examined, and the basic design concept to achieve small excess loss along with high bending loss was presented. The desired attenuation could be obtained easily by the controlled bending of the tapered fiber. The insertion loss of the fabricated variable optical attenuator was $0.2 \mathrm{~dB}$ and the dynamic range for the attenuation was $35 \mathrm{~dB}$. The simple structure, simple fabrication procedure, and easy control of the attenuation level of the proposed device can be used to develop a low cost and small size VOA for optical metro and access networks.

\section{REFERENCES}

1. S. S. Lee, Y. S. Jin, and T. K. Yoo, "Polymeric tunable optical attenuator with an optical monitoring tap for WDM transmission network," IEEE Photon. Technol. Lett. 11, 590-592 (1999).

2. Y. O. Noh, C. L. Lee, J. M. Kim, W. Y. Hwang, Y. H. Won, H. J. Lee, S. G. Han, and M. C. Oh, "Polymer waveguide variable optical attenuator and its reliability," Opt. Comm. 242, 533-540 (2004).

3. T. Kawai, M. Koga, M. Okuno, and T. Kitoh, "PLC type compact variable optical attenuator for photonic transport network," Electron. Lett. 34, 264-265 (1998).

4. J. H. Lee, Y. Y. Kim, S. S. Yun, H. Kwon, Y. S. Hong, J. H. Lee, and S. C. Jung, "Design and characteristics of a micromachined variable optical attenuator with a silicon wedge," Opt. Comm. 221, 323-330 (2003).

5. C. Marxer, P. Ggriss, and N. F. de Rooij, "A variable optical attenuator based on silicon micromechanics," IEEE Photon. Technol. Lett. 11, 233-235 (1999).

6. N. A. Riza and S. Sumriddetechkajorn, "Digitally controlled fault-tolerant muliwavelength programmable fiber optic attenuator using a two dimensional digital micromirror devices," Opt. Lett. 24, 83-84 (1999).

7. J. Villatoro, A. Diez, J. L. Cruz, and M. V. Andres, "In-line highly sensitive hydrogen sensor based on palladiumcoated single-mode tapered fibers," IEEE Sensors Journal 3, 533-537 (2003).

8. C. Bariain, I. R. Matas, F. J. Arregui, and M. Lopez-
Amo, "Optical fiber humidity sensor based on a tapered fiber coated with agarose gel," Sens. and Actuators B: Chemical 69, 127-131 (2000).

9. V. Morozove, H. Fan, L. Eldada, L. Yang, and Y. Shi, "Fused fiber-optic variable attenuator," in Proc. Optical Fiber Communication Conference (Baltimore, USA, Mar. 2000), pp. 22-24.

10. A. Diez, M. V. Andres, and D. O. Culverhouse, "In-line polarizers and filters made of metal-coated tapered fibers: resonant excitation of hybrid plasma modes," IEEE Photon. Technol. Lett. 10, 833-835 (1998).

11. J. Villatoro, D. Monzon-Hernandez, and D. Luna-Moreno, "In-line tunable band-edge filter based on a single-mode tapered fiber coated with a dispersive material," IEEE Photon. Technol. Lett. 17, 1665-1667 (2005).

12. W. A. Gambling, H. Matsumura, and C. M. Ragdale, "Curvature and microbending losses in single mode optical fibers,” Optical and Quantum Electronics 11, 43-59 (1979).

13. L. C. Bobb, P. M. Shanker, and H. D. Krumboltz, "Bending effects in biconically tapered single mode fibers,” J. Lightwave Technol. 8, 1084-1090 (1990).

14. P. M. Shankar, L. C. Bobb, and H. D. Krumboltz, "Coupling of modes in bent biconically tapered single-mode fibers," J. Lightwave Technol. 9, 832-837 (1991).

15. C. Bariain, I. R. Matias, and F. J. Francisco, "Tapered optical-fiber-based pressure sensor," Opt. Eng. 39, 2241-2247 (2000).

16. I. R. Matias, M. Lopez-Amo, and F. Mantero, "Low-cost optical amplitude modulator based on a tapered single-mode optical fiber," Appl. Opt. 40, 228-234 (2001).

17. D. H. Lee, K. H Kwon, J. W. Song, and J. H. Park, "Variable optical fiber attenuator using bending-sensitive fiber,” J. Opt. Soc. Korea 8, 83-89 (2004).

18. T. A. Birks and Y. W. Li, "The shape of fiber tapers," J. Lightwave Technol. 10, 432-438 (1992).

19. R. P. Kenny, T. A. Birks, and K. P. Oakley, "Control of optical fiber taper shape," Electron. Lett. 27, 1654-1656 (1991).

20. J. Villatoro, D. Monzon-Hernandez, and E. Mejia, "Fabrication and modeling of uniform waist single-mode tapered optical fiber sensor,” Appl. Opt. 42, 2278-2283 (2003). 